\title{
Modeling and optimization of lipase-catalyzed synthesis of dilauryl adipate ester by response surface methodology
}

\begin{abstract}
BACKGROUND: Adipate esters are used as low-temperature and low-viscosity plasticizers for polyvinyl chloride and its copolymers. In this work, optimization of lipase-catalyzed production of dilauryl adipate was carried out using response surface methodology (RSM) based on a four-factor-five-level central composite rotatable design (CCRD). Immobilized lipase from Candida antarctica (Novozym 435) was used as catalyst in this reaction. Various reaction parameters affecting the synthesis of adipate ester, including alcohol/acidmolar ratio, amount of enzyme, temperature and reaction time, were investigated. RESULTS: Statistical analysis showed that the amount of enzyme was less significant than the other three factors. The optimal conditions for the enzymatic reaction were obtained at 5.7:1 substrate molar ratio using $0.18 \mathrm{~g}$ of enzyme at $53.1{ }^{\circ} \mathrm{C}$ for $282.2 \mathrm{~min}$. Under these conditions the esterification percentage was $96.0 \%$. CONCLUSIONS: The results demonstrated that response surface methodology can be applied effectively to optimize the lipase-catalyzed synthesis of adipate ester. The optimum conditions can obtained be used to scale up the process.
\end{abstract}

Keyword: lipase, esterification, adipate ester, response surface methodology, central composite rotatable design 